\title{
Synergistic effects of extracorporeal shockwave therapy and modified Ojayeonjonghwan on erectile dysfunction in an animal model of diabetes
}

\author{
Hyun Cheol Jeong ${ }^{1, *}$, Woong Jin Bae ${ }^{2,3, *}$, Guan Qun Zhu' ${ }^{2}$, Seung Hwan Jeon ${ }^{2}$, Sae Woong Choi ${ }^{2}$, Su Jin Kim², \\ Hyuk Jin $\mathrm{Cho}^{2}$, Sung-Hoo Hong ${ }^{2}$, Ji Youl Lee ${ }^{2}$, Sung Yeoun Hwang ${ }^{4}$, Sae Woong Kim ${ }^{2,3}$ \\ 'Department of Urology, Kangdong Sacred Heart Hospital, Hallym University College of Medicine, Seoul, ${ }^{2}$ Department of Urology, College of Medicine, The Catholic \\ University of Korea, Seoul, ${ }^{3}$ Catholic Integrative Medicine Research Institute, College of Medicine, The Catholic University of Korea, Seoul, ${ }^{4}$ KEMIMEDI, Seoul, Korea
}

Purpose: We investigated the synergy effect of extracorporeal shock wave therapy (ESWT) with modified Ojayeonjonghwan (Korean herbal formula, $\mathrm{KH}-204$ ) in an animal model of diabetes mellitus (DM)-induced erectile dysfunction (ED).

Materials and Methods: Eight-week-old male Sprague-Dawley rats were used in this study. DM was induced by an intraperitoneal injection of streptozotocin. DM rats were divided into 5 groups ( $n=10$ per group): group 1, control; group 2, DM; group 3, DM+ESWT; group 4, DM+KH-204; and group 5, DM+ESWT+KH-204. In ESWT groups, rats were treated with ESWT at the penis 3 times a week for 2 weeks under anesthesia. The $\mathrm{KH}-204$ groups were treated with a daily oral dose of $\mathrm{KH}-204$ for 12 weeks. After all treatments, intracavernosal pressure (ICP) was measured, and the cavernous tissues were evaluated by Masson's trichrome, immunohistochemistry and western blot analysis.

Results: ICP was evaluated as a measurement of erectile function. The DM+ESWT, DM+KH-204, and DM+ESWT+KH-204 groups showed significantly restored erectile function compared with the DM group $(p<0.05)$. Among these groups, the DM+ESWT+KH-204 group showed the highest ICP. Moreover, ESWT and KH-204 treatment restored smooth muscle contents and many parameters related to potency (vascular endothelial growth factor, neuronal nitric oxide synthase (NOS), endothelial [NOS] and platelet endothelial cell adhesion molecule-1) compared with the DM group $(p<0.05)$.

Conclusions: We confirmed the potential efficacy of ESWT and KH-204 in the treatment of ED patients using an animal model. The combination treatment of $\mathrm{KH}-204$ and ESWT is expected to have good potential clinical results in the future treatment of refractory ED.

Keywords: Animals; Erectile dysfunction; Extracorporeal shockwave therapy; Herbal medicine

This is an Open Access article distributed under the terms of the Creative Commons Attribution Non-Commercial License (http://creativecommons.org/licenses/by-nc/4.0) which permits unrestricted non-commercial use, distribution, and reproduction in any medium, provided the original work is properly cited.

Received: 14 March, 2019 - Accepted: 2 May, 2019

Corresponding Author: Sae Woong Kim

Department of Urology, Seoul St. Mary's Hospital, College of Medicine, The Catholic University of Korea, 222 Banpo-daero, Seocho-gu, Seoul 06591, Korea TEL: +82-2-2258-6226, FAX: +82-2-599-7839, E-mail: ksw1227@catholic.ac.kr

ORCID: https://orcid.org/0000-0002-9127-9400

*These authors contributed equally to this study and should be considered co-first authors. 


\section{INTRODUCTION}

Erectile dysfunction (ED) is a chronic disease that lowers an individual's quality of life [1]. Phosphodiesterase 5 inhibitors (PDE5I) are one of the first-line drugs for ED treatment [2]. However, these medications merely improve ED symptoms rather than change the pathophysiologic derangements of $\mathrm{ED}$, such as distal vessel disorder caused by diabetes mellitus (DM), corpus cavernosum damage due to trauma and nerve injury resulting from radical prostatectomy [3]. In recent years, low-intensity extracorporeal shock wave therapy (ESWT) has been widely used with exciting results in the medical domain as a novel and promising approach for a range of conditions, including, for example musculoskeletal disorders [4], myocardial infarction [5], and non-healing wounds [6].

In urology, ESWT has been used not only for Peyronie's disease and chronic pelvic pain but also for ED [7]. Vardi et al. [8] recorded detailed instructions about the ESWT approach as early as 2010. Over the next several years, many researchers published their studies using ESWT as an ED treatment, in which the major results showed that ESWT had a positive influence on $\operatorname{ED~[9,10].~In~our~previous~study,~}$ we found ESWT could increase the expression of vascular endothelial growth factor (VEGF) in the corpus cavernosum, thereby promoting the repair of injured tissue [11,12].

Peripheral vascular injury caused by chronic hyperglycemia in the corpus cavernosum is an important cause of DM induced ED [13]. Meanwhile, oxidative stress plays a key role in vascular injury induced ED [14]. In our previous study in an animal model, we found that modified Ojayeonjonghwan (Korean herbal formula, KH-204), an oriental herbal medicine decreased oxidative stress $[15,16]$. Thus, we believe that KH-204 may, at least in part, promote the repair of injured penile tissue.

This study was designed to investigate the application of the combination of ESWT and KH-204 for ED. Our goal was to obtain authentic data to determine the efficacy of the combination ESWT and KH-204 in the treatment of ED.

\section{MATERIALS AND METHODS}

\section{Experimental animals and study design}

Eight-week-old male Sprague-Dawley rats weighing 270 to $300 \mathrm{~g}$ (Orient Bio Co., Seongnam, Korea) were used in this study. DM was induced by an intraperitoneal injection of streptozotocin $(50 \mathrm{mg} / \mathrm{kg}$ in sodium citrate buffer, $\mathrm{pH}$ 4.5). After 72 hours, DM was confirmed by blood glucose levels greater than $300 \mathrm{mg} / \mathrm{dL}$ determined by Accu-Check
Active blood glucose monitor (Hoffmann-La Roche Ltd, Basel, Switzerland), and blood samples were obtained from tail veins. The rats were randomly divided into 5 groups ( $\mathrm{n}=10$ per group): normal, DM, DM+ESWT, DM+KH-204, and DM+ESWT+KH-204. After 12 weeks, all rats underwent intracavernosal pressure (ICP) measurement and were sacrificed under anesthesia after treatments. Rats were anesthetized with an intraperitoneal injection of tiletamine and zolazepam $(30 \mathrm{mg} / \mathrm{kg})$ and xylazine $(10 \mathrm{mg} / \mathrm{kg})$.

\section{Preparation of a Korean herbal formula (KH-204)}

The major ingredients in KH-204 include 5 plants, as previously described: Cornus officinalis (32\%), Lycium chinense (32\%), Rubus coreanus (16\%), Cuscuta chinensis (16\%), and Schisandra chinensis (4\%) [17]. It was developed by a company that makes oriental herbal medicines, KEMIMEDI Co, Ltd. (Seoul, Korea). The quality of each extract was confirmed using high-performance liquid chromatography (HPLC) as previously described [17]. The marker compounds of $C$. officinalis, $L$. chinense, $R$. coreanus, $C$. chinensis, and S. chinensis are loganin, betaine, ellagic acid, hyperoside, and schizandrin in the HPLC chromatogram, respectively. Each herb $(20 \mathrm{~kg})$ was extracted in $200 \mathrm{~L}$ of distilled $30 \%$ ethanol and refluxed at $98^{\circ} \mathrm{C} \pm 2^{\circ} \mathrm{C}$ for 3 hours. The extract was filtered, and the liquid from the filtrates was removed by a rotary evaporator and a spray dryer. $\mathrm{KH}-$ 204 was dissolved in distilled water and administered orally through an $8 \mathrm{~F}$ red Rob-Nel catheter once a day for 4 weeks.

\section{ESWT treatment}

After 4 weeks of DM induction, rats in the DM+ESWT, and DM+ESWT+KH-204 groups underwent MT2000H shockwave treatment (Urontech, Hwaseong, Korea). Under anesthesia, each rat was placed in the supine position, and shaved, and the prepuce was degloved. Ultrasound gel was applied to the penis and a shockwave applicator was placed at the penis. A total of 300 shocks were delivered at an energy level of $0.1 \mathrm{~mJ} / \mathrm{mm}^{2}$ and a frequency of 120 shocks/ min. This procedure was performed 3 times a week for 2 weeks, which is similar to the treatment course used for patients with ED in clinical practice. In DM+ESWT+KH-204 group, ESWT and KH-204 treatment were started together.

\section{ICP measurement}

Erectile function was assessed 1 week after the treatment course for all 5 groups. After placing the rat in the supine position, the corpus cavernosum, crus of the penis and the pelvic ganglion were exposed through abdominal incision.

The carotid artery and cavernous nerve were exposed 
to detect the mean arterial pressure (MAP) and ICP. BD Intramedic PE-50 tubing (BD, Franklin Lakes, NJ, USA) was inserted into the carotid artery to measure the MAP. At the same time, a 23-gauge butterfly needle filled with heparin was inserted in the corpus cavernosum, and then connected to a pressure transducer (Grass model S48K, Grass Instrument Division; Astro-Med Inc., West Warwick, RI, USA) to measure the ICP. A bipolar electrical stimulator was used to stimulate the pelvic ganglion and the cavernosal nerve for 50 seconds at $10 \mathrm{~V}$ and 0.5 milliseconds at $2.4 \mathrm{~mA}$, respectively. ICP and MAP were recorded by a pressure transducer (Grass Stimulator Recorder, Grass model S48K, Grass Instrument Division). The maximal ICP was calculated and recorded on a computer with a PowerLab commercial data acquisition system (AD Instruments, Dunedin, New Zealand). BD Intramedic PE-50 tubing was used to measure MAP. The ICP/MAP ratio and ratio of area under the curve to MAP was measured to assess erectile function. After the stimulation test, the penis was harvested from each rat. Samples were fixed in $4 \%$ paraformaldehyde for 24 hours at $4^{\circ} \mathrm{C}$ before paraffin blocks were created [18].

\section{Masson's trichrome staining}

Masson's trichrome staining was performed on corporal tissue sections. The middle portions of the penile samples were fixed overnight in $4 \%$ formalin, washed, and stored in $70 \%$ alcohol at $4^{\circ} \mathrm{C}$ until processing for paraffin-embedded tissue sectioning $(4 \mu \mathrm{m})$. After staining, the color distribution in the tissue was measured using Image Pro Plus version 5.0 (Media Cybernetics, Silver Spring, MD, USA). After the entire color distribution of the image was calculated, the muscle tissue was expressed using the color blue. The mean ratio of collagen and muscle fiber was calculated.

\section{Histochemistry}

The following primary antibodies were used: neuronspecific $\beta$-III tubulin (diluted 1:200, Abcam, Cambridge, UK), neuronal nitric oxide synthase (nNOS, diluted 1:200; Santa Cruz Biotechnologies, Santa Cruz, CA, USA), a-smooth muscle actin ( $\alpha$-SMA, diluted 1:500; Abcam), VEGF (diluted 1:200; Santa Cruz Biotechnologies) and 6-diamidino-2-phenylindole (DAPI; Vector Laboratories Inc, Burlingame, CA, USA) was used to stain nuclei. Digital images were obtained using a Zeiss LSM 510 Meta confocal microscope (Zeiss, Oberkochen, Germany), and the mean intensity was calculated using ZEN 2012 (Zeiss).

\section{Western blot analysis of endothelial NOS and platelet endothelial cell adhesion molecule-1 expression}

Corpus cavernosum tissue was homogenized using RIPA buffer (Cell Signaling Technology, Danvers, MA, USA) containing an ethylenediaminetetra acetic acid-free protease inhibitor cocktail and a phosphatase inhibitor cocktail (Roche Diagnostics GmbH, Basel, Switzerland). The resultant lysates were clarified by centrifugation at $12,000 \times g$ for 10 minutes at $4^{\circ} \mathrm{C}$ and their supernatants extracted. Proteins were electrophoresed on NuPAGE $4 \%$ to $12 \%$ Bis-Tris gels (Invitrogen, Carlsbad, CA, USA) and transferred onto nitrocellulose membranes. The membranes were incubated with primary antibodies against platelet endothelial cell adhesion molecule-1 (PECAM-1, diluted 1:500; Abcam), endothelial NOS (eNOS, ab5589, diluted 1:1,000; Abcam), or phosphorylated eNOS (P-eNOS, Ser1177, diluted 1:500; Cell Signaling Technology). Next, the membranes were incubated with secondary antibodies conjugated to horseradish peroxidase for 1 hour at room temperature. Enhanced chemiluminescence reagents (Amersham, Arlington Heights, IL, USA) were used for densitometric analysis of band intensity.

\section{Statistical analysis}

The number of animals planned by the researchers is a statistically minimal number when reference is made to papers on animal experimental results similar to this experiment. Fifty rats were divided into groups according to the failure rate of diabetic model induction (about 30\%, the probability of no diabetes induced by streptozotocin administration). All data are presented as means \pm standard deviations (SDs). Data were analyzed using IBM SPSS Statistics ver. 22.0 (IBM Co., Armonk, NY, USA). Data are expressed as means \pm standard errors. Results from different groups were compared using the Mann-Whitney U test. A p-value less than 0.05 was considered to be statistically significant.

\section{Ethics statement}

All animal experiments in this study were approved by the Institutional Animal Care and Use Committee of The Catholic University of Korea (approval number: CUMC-20160310-01).

\section{RESULTS}

\section{ICP measurement for improvement of ED}

The DM group showed statistically significantly lower 
A
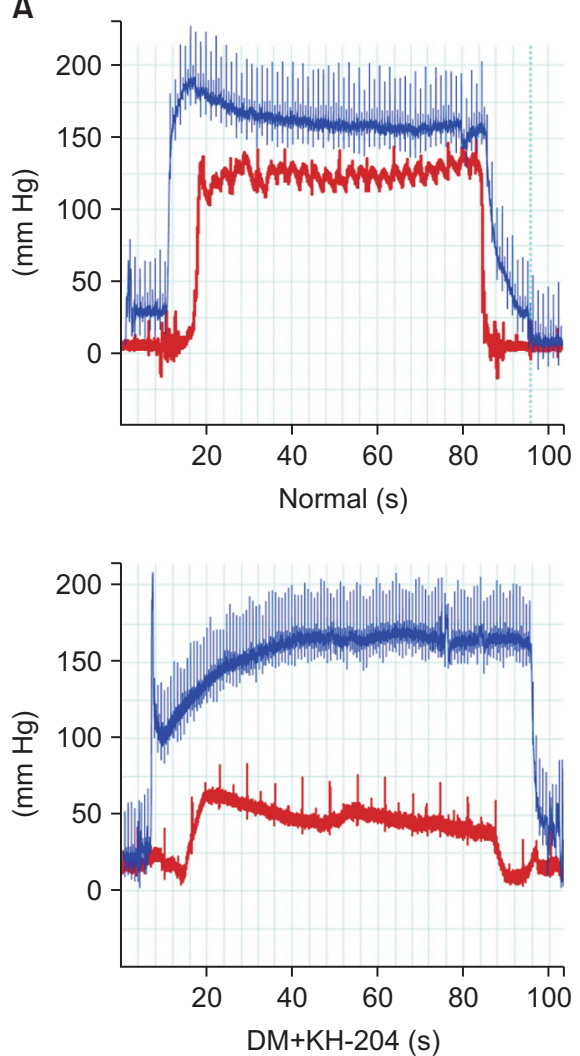
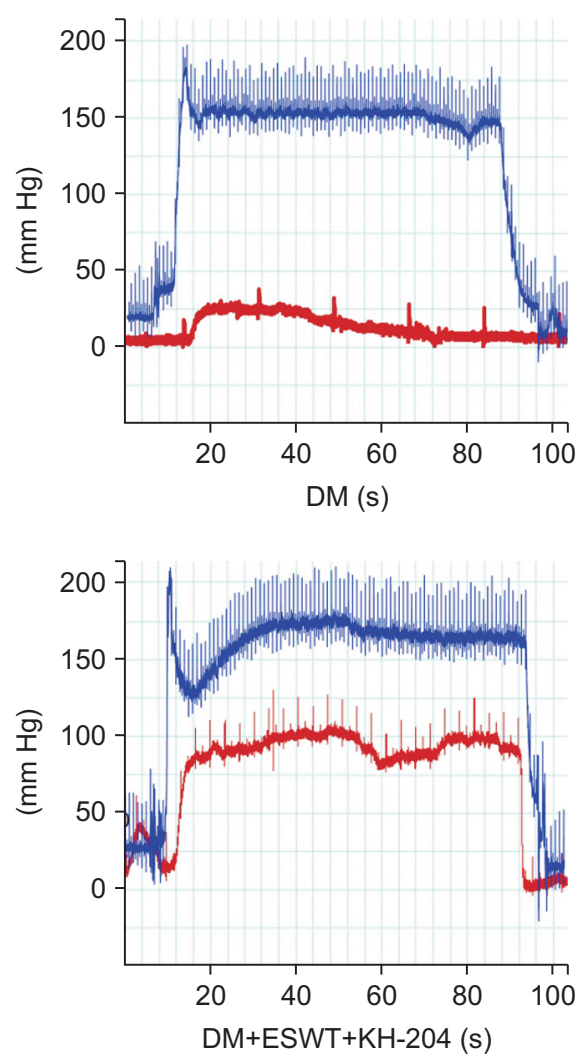

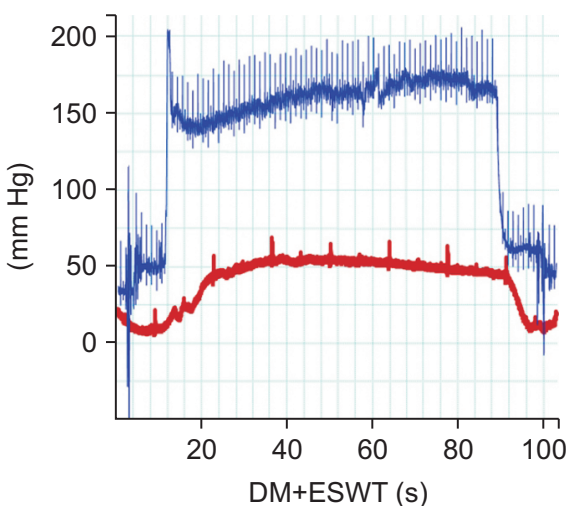

B

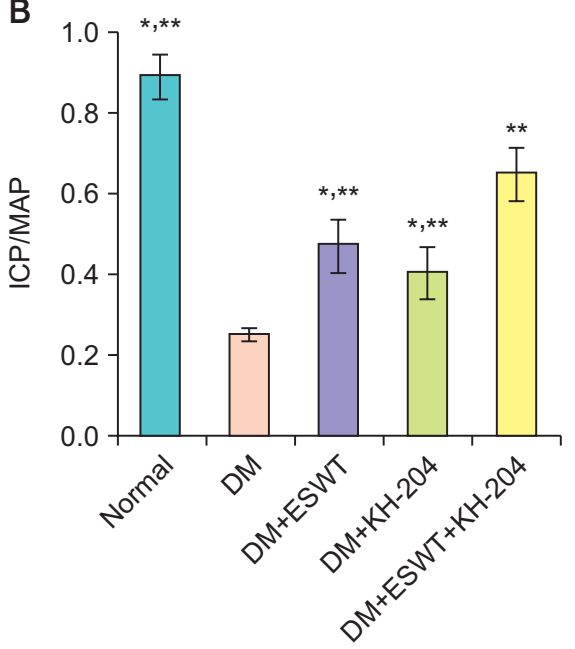

Fig. 1. Intracavernosal pressure (ICP), ICP/mean arterial pressure (MAP) of each groups. (A) Representative ICP recordings in response to cavernous nerve stimulation. (B) ICP/MAP ratios after nerve stimulation of the different groups. DM, diabetes mellitus; ESWT, extracorporeal shock wave therapy; $\mathrm{KH}$, Korean herbal formula. ICP: red line, MAP: blue line. Data are expressed as means \pm standard deviations. *Statistical significance in comparison with the $\mathrm{DM}+\mathrm{ESWT}+\mathrm{KH}-204$ group. ${ }^{*}$ Statistical significance in comparison with the DM group.

ICP values than control group, but showed significant recovery in the ESWT and KH-204 groups (Fig. 1). The mean ICP/MAP ratios for the control, DM, DM+ESWT, $\mathrm{DM}+\mathrm{KH}-204$, and DM+ESWT+KH-204 groups were $0.88 \pm 0.13$, $0.25 \pm 0.04,0.47 \pm 0.16,0.40 \pm 0.15$, and $0.64 \pm 0.15$ respectively. The mean ratio was significantly lower in the DM group than in the control group ( $p<0.001)$, but recovered in the ESWT+KH-204 treated group compared with the DM group significantly ( $p=0.003$ ). There were no statistical differences between the ratios of the ESWT and KH-204 single treated groups. The ESWT+KH-204 group showed a higher ratio compared with the ESWT and KH-204 single treatment groups ( $\mathrm{p}=0.042$ and $\mathrm{p}=0.049$ respectively).

\section{ESWT+KH-204 effects on stimulation of smooth muscle/collagen ratio \\ The DM group showed statistically significantly less corporal smooth muscle tissue and higher collagen deposition than the control group (Fig. 2). The mean muscle/collagen}

ratios \pm SDs for the control, DM, DM+ESWT, DM+KH-204, and DM+ESWT+KH-204 groups were $0.21 \pm 0.04,0.06 \pm 0.02$, $0.10 \pm 0.02,0.09 \pm 0.03$, and $0.15 \pm 0.05$ respectively. The mean ratio was significantly lower in the DM group than in the control group ( $p<0.001$ ), but it recovered in the ESWT+KH-204 treated group compared with the DM group $(\mathrm{p}=0.003)$. The rats treated with ESWT+KH-204 showed a higher ratio than the single treatment groups $(p=0.044$ and $p=0.048$ respectively).

As shown in Fig. 3, $\alpha_{-}$SMA expression levels were elevated in the corpus cavernosum, indicating that smooth muscle contents in corpus cavernosal tissues. The mean intensities \pm SDs $\alpha_{-}$SMA of -positive areas for the control, DM, DM+ESWT, DM+KH-204, and DM+ESWT+KH-204 groups were $296.86 \pm 78.40,80.55 \pm 30.77,180.58 \pm 73.13,147.92 \pm 48.34$, and $249.61 \pm 75.01$ respectively. 


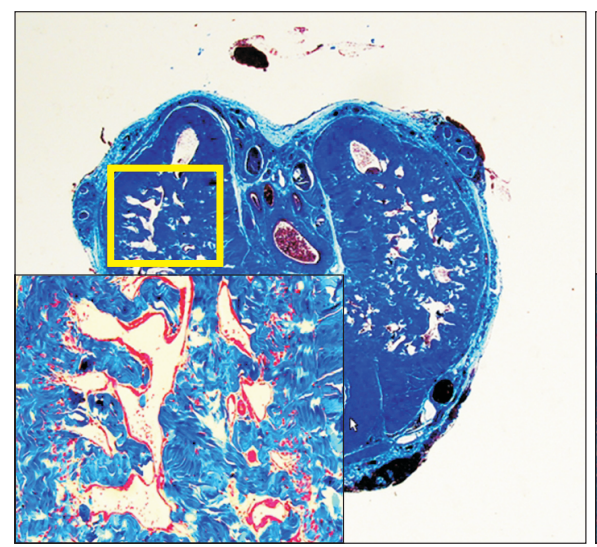

Normal



$\mathrm{DM}+\mathrm{KH}-204$



DM



$\mathrm{DM}+\mathrm{ESWT}+\mathrm{KH}-204$

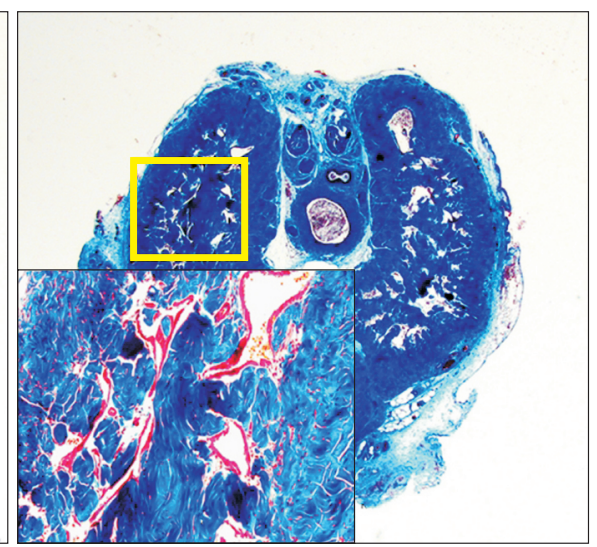

DM+ESWT

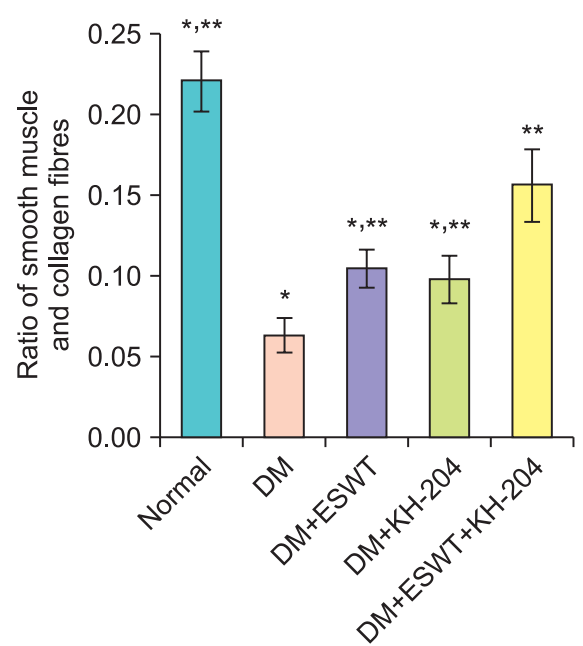

Fig. 2. Results of smooth muscle content evaluation by Masson's trichrome staining. Mean of the smooth muscle and collagen fiber ratios of the different groups (graph) ( $\times 40$; inset, $\times 100)$. DM, diabetes mellitus; ESWT, extracorporeal shock wave therapy; KH, Korean herbal formula. Data are expressed as means \pm standard deviations. *Statistical significance in comparison with the DM+ESWT+KH-204 group. ${ }^{* *}$ Statistical significance in comparison with the DM group.

\section{ESWT+KH-204 results in more VEGF expression in corporal tissue}

We performed immunohistochemical (IHC) staining to evaluate the expression of VEGF in corporal tissue (Fig. 4A). The mean intensities of VEGF staining for the control, $\mathrm{DM}, \mathrm{DM}+\mathrm{ESWT}, \mathrm{DM}+\mathrm{KH}-204$, and DM+ESWT+KH-204 groups were $79.39 \pm 8.33,30.29 \pm 9.43,72.75 \pm 20.45,65.82 \pm 11.61$, and $89.22 \pm 14.35$ respectively. It was decreased significantly in the DM group compared with the control group ( $\mathrm{p}<0.001)$, while the ESWT+KH-204 group restored VEGF staining compared with the DM group significantly $(\mathrm{p}<0.001)$. The ESWT+KH-204 combined group showed higher intensities compared with the single-treatment groups $(\mathrm{p}=0.011$ and $\mathrm{p}=0.031$ respectively).

\section{ESWT+KH-204 activates nNOS expression in the dorsal penile nerve}

nNOS expression was assessed by IHC staining in dorsal nerve (Fig. 4B). In the DM group, rats showed a statistically significant decreased amount of nNOS-containing nerves. The mean intensities \pm SDs of nNOS-positive areas for the control, DM, DM+ESWT, DM+KH-204, and DM+ESWT+ $\mathrm{KH}-204$ groups were $366.16 \pm 60.95,83.45 \pm 21.64,210.82 \pm 54.16$, $161.48 \pm 26.68$, and $239.18 \pm 50.01$ respectively (Fig. 4B). nNOS expression was significantly decreased in the DM group but it was significantly greater in the ESWT+KH-204 treatment group than in the DM group ( $\mathrm{p}<0.001$ ). The ESWT group recovered significantly higher nNOS levels as compared with the KH-204 group $(p=0.045)$. The combined treatment group showed higher levels than the single treatment groups ( $\mathrm{p}=0.039$ and $\mathrm{p}=0.01$ respectively).

\section{ESWT+KH-204 promotes endothelial regenera- tion in corporal tissue}

eNOS and PECAM-1 expression levels were assessed in corporal tissue by western blotting. P-eNOS expression was 




Normal



$\mathrm{DM}+\mathrm{KH}-204$

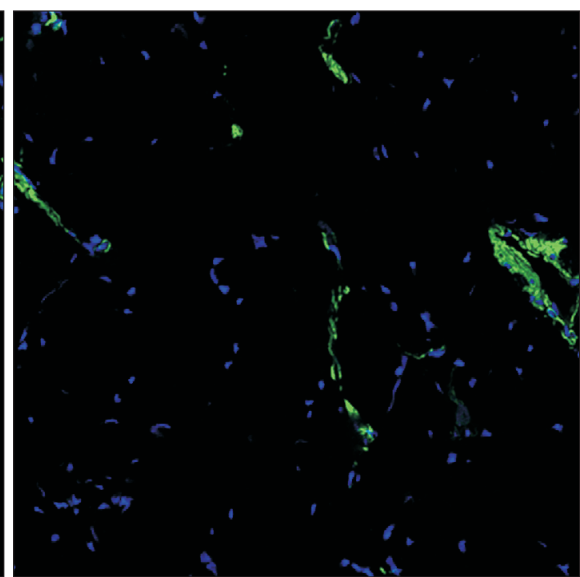

DM

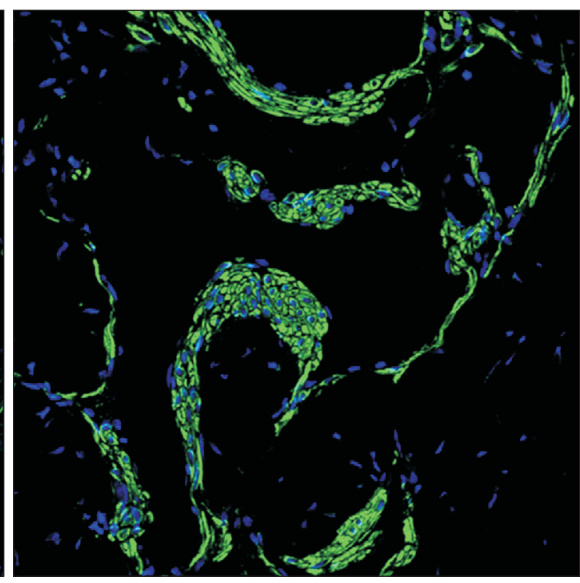

$\mathrm{DM}+\mathrm{ESWT}+\mathrm{KH}-204$

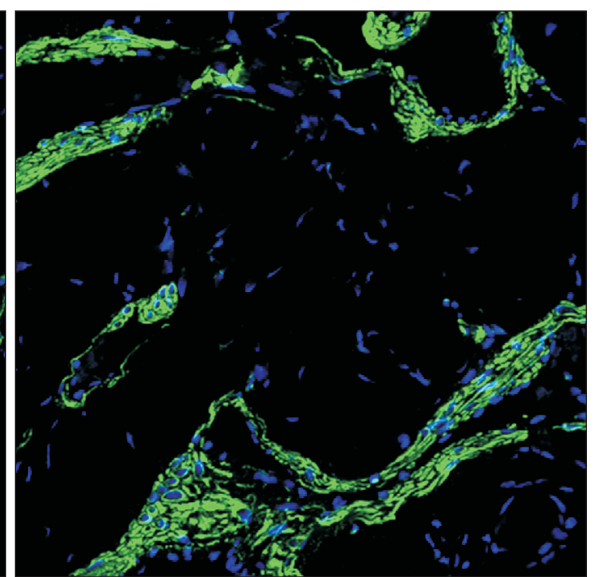

DM+ESWT



Fig. 3. Results of smooth muscle content evaluation by $\alpha$-smooth muscle actin ( $\alpha$-SMA). Mean of the smooth muscle and collagen fiber ratios of the different groups (graph). DM, diabetes mellitus; ESWT, extracorporeal shock wave therapy; KH, Korean herbal formula. Data are expressed as means \pm standard deviations. *Statistical significance in comparison with the DM+ESWT+KH-204 group. ${ }^{*}$ Statistical significance in comparison with the DM group.

significantly lower in the DM group compared with the control group $(p<0.001)$ and it was significantly restored with ESWT+KH-204 treatment $(p=0.001)$. The mean densities of P-eNOS/eNOS for the control, DM, DM+ESWT, DM+KH-204, and DM+ESWT+KH-204 groups were $0.34 \pm 0.04,0.14 \pm 0.03$, $0.22 \pm 0.07,0.23 \pm 0.06$, and $0.30 \pm 0.05$ respectively (Fig. $5 \mathrm{~A}$ ). The mean densities of the PECAM-1/ $\beta$-actin ratio for the control, DM, DM+ESWT, DM+KH-204, and DM+ESWT+KH-204 groups were $0.34 \pm 0.05,0.10 \pm 0.03,0.20 \pm 0.06,0.18 \pm 0.05$, and $0.28 \pm 0.04$ respectively (Fig. 5B). The ESWT+KH-204 treated group showed significantly restored PECAM-1 expression compared with DM rats $(p<0.001)$. The combination treatment group showed higher densities and ratios compared with the single treatment groups (ESWT and KH-204; $p=0.007$ and $p=0.02$ respectively) in PECAM-1/ $\beta$-actin ratio.

\section{DISCUSSION}

The use of ESWT for the clinical treatment of ED has been gradually increasing in recent years. Even though most studies showed that ESWT had a positive effect on $\mathrm{ED}, \mathrm{Lu}$ et al. [19] found that ESWT was more effective in mild ED. From a series of randomized sham-controlled trials, some researchers obtained positive results that the ESWT approach showed significantly good results in ED related questionnaire, even in PDE5I non-responders [20]. As a first-line drug for ED, PDE5I still has some limitations. In addition to non-responses, side-effects such as headache, dyspepsia, facial flushing, nasal congestion, and backache, cannot be ignored by patients and urologists and patients with heart disease have limited use [21]. Moreover PED5I have reduced efficacy in patients with diabetic ED, and is not a fundamental treatment and requires continuous use [22]. The mechanism of ESWT has not yet been fully 
A

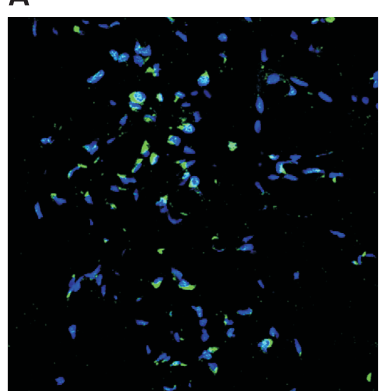

Normal

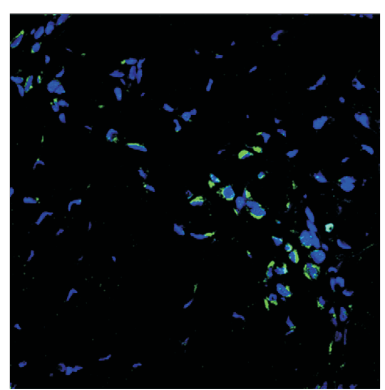

$\mathrm{DM}+\mathrm{KH}-204$

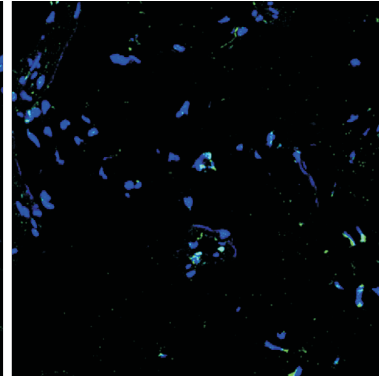

DM

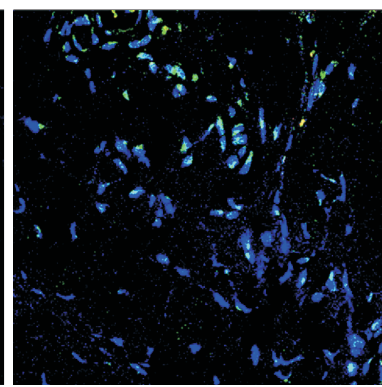

$\mathrm{DM}+\mathrm{ESWT}+\mathrm{KH}-204$

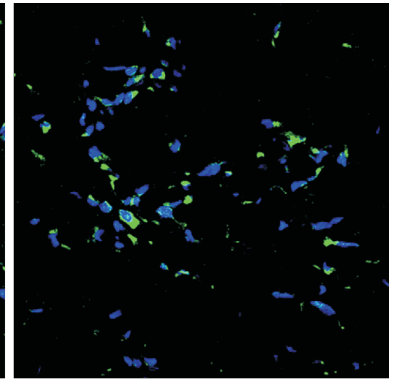

DM+ESWT

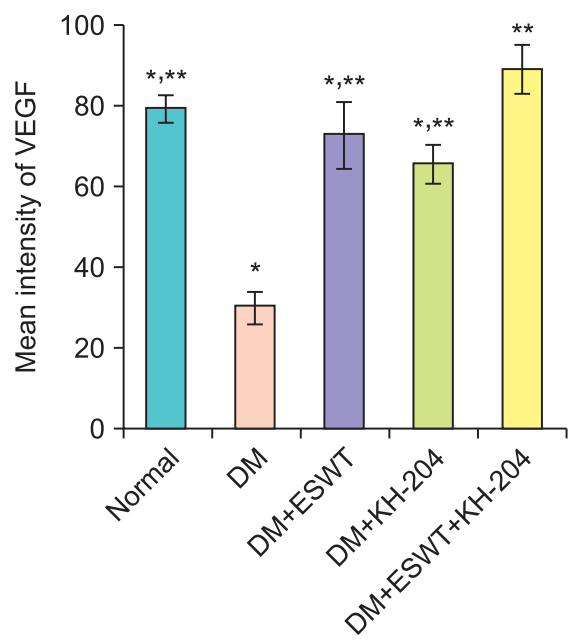

B

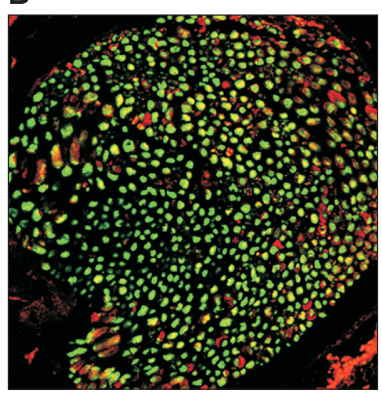

Normal

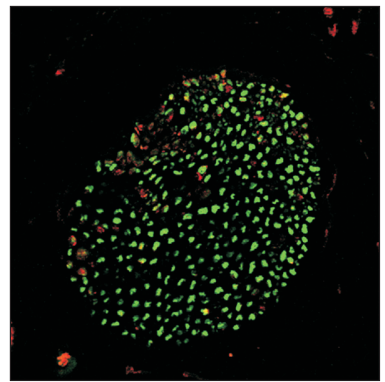

$\mathrm{DM}+\mathrm{KH}-204$

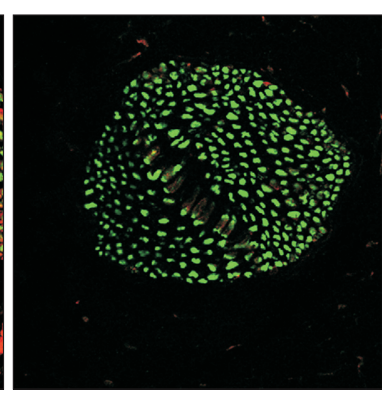

DM

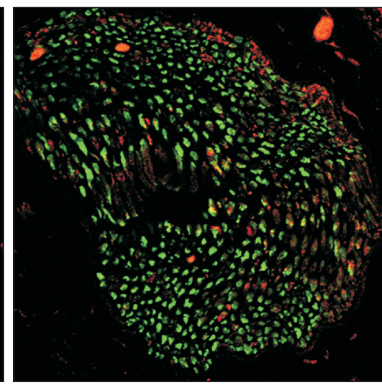

$\mathrm{DM}+\mathrm{ESWT}+\mathrm{KH}-204$

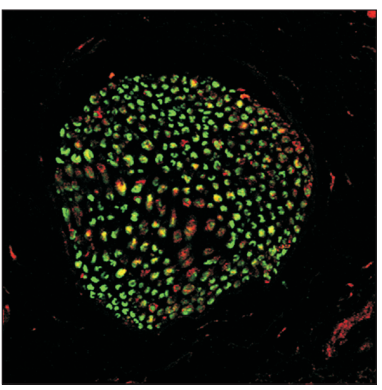

DM+ESWT

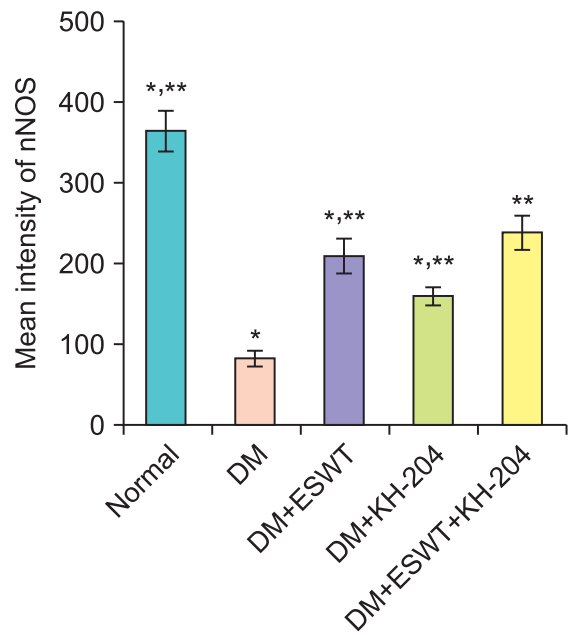

Fig. 4. (A) Immunohistochemical (IHC) staining of vascular endothelial growth factor (VEGF) expression in the corpus cavernosum of each group. Mean intensities of VEGF expression in the different groups are represented in the graphs. (B) IHC staining of neuronal nitric oxide synthase (nNOS) expression in the dorsal penile nerve of each group. Mean intensities of nNOS expression levels of the different groups are represented in the graphs. DM, diabetes mellitus; ESWT, extracorporeal shock wave therapy; KH, Korean herbal formula. Data are expressed as means \pm standard deviations. *Statistical significance in comparison with the DM+ESWT+KH-204 group. ${ }^{*}$ Statistical significance in comparison with the DM group.

elucidated, but there are several hypotheses. The shear stress of ESWT induces endothelial proliferation due to intra and extracellular responses. The increase in eNOS and VEGF due to ESWT is known to promote angiogenesis and restore $\mathrm{ED}$ [23]. In addition, ESWT is reported to help recruitment of stem cells, which is thought to restore the ED by helping the recovery of the nerve and blood vessels of erectile tissues [24].

Gerber et al. [25] believed that one main cause for DMinduced ED was vascular injury in the corpus cavernosum due to chronic hyperglycemia. And distal vessel spasm in the corpus cavernosum causes penis tissue ischemia. Jeon et al. [11] found that in the corpus cavernosum of rats with 
A
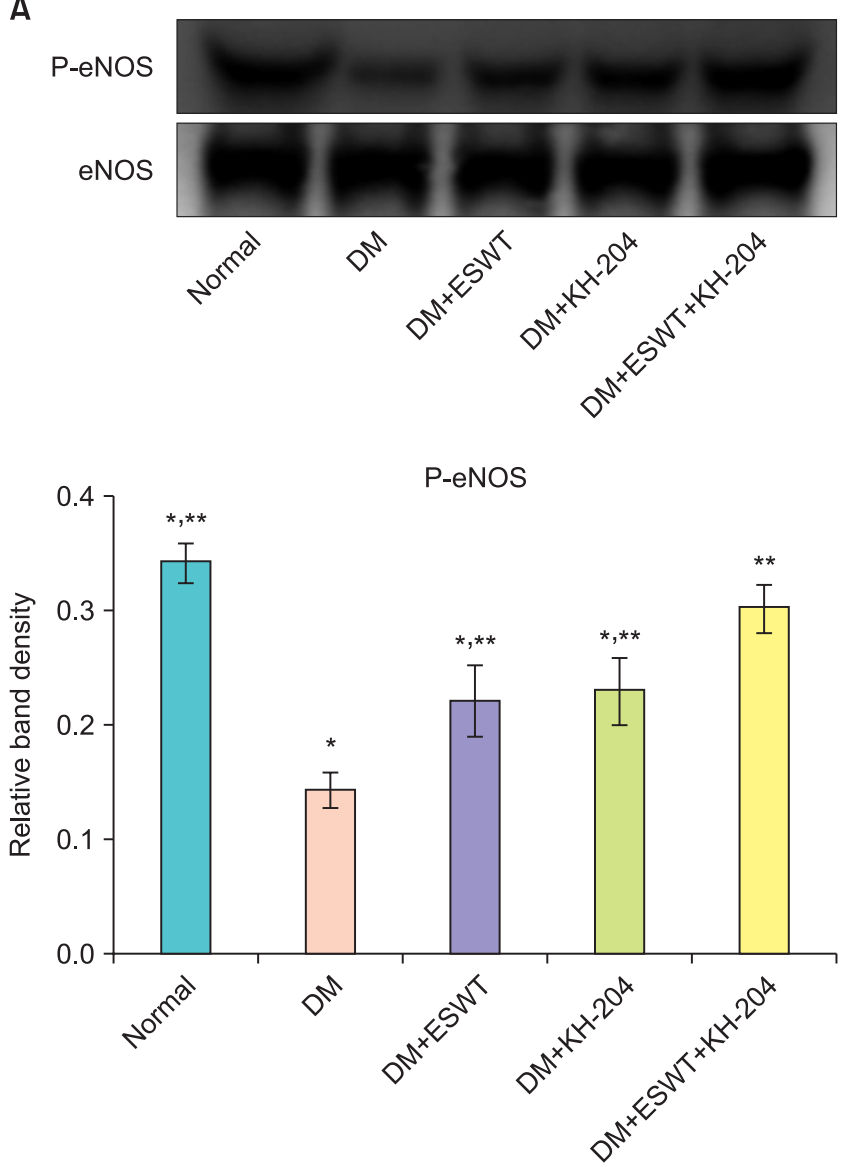

B

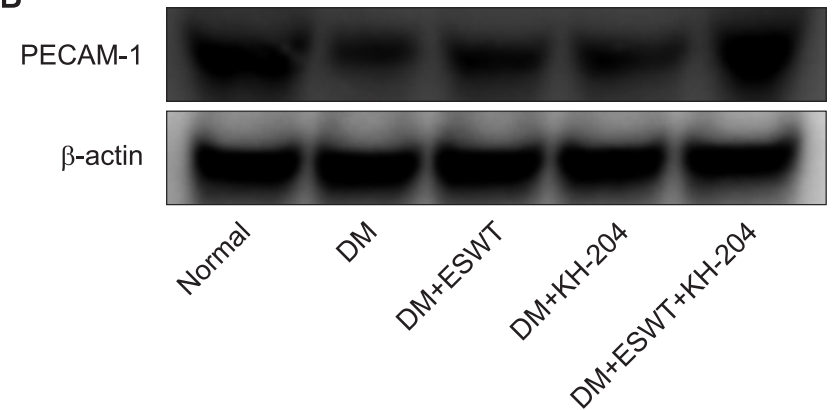

PECAM-1

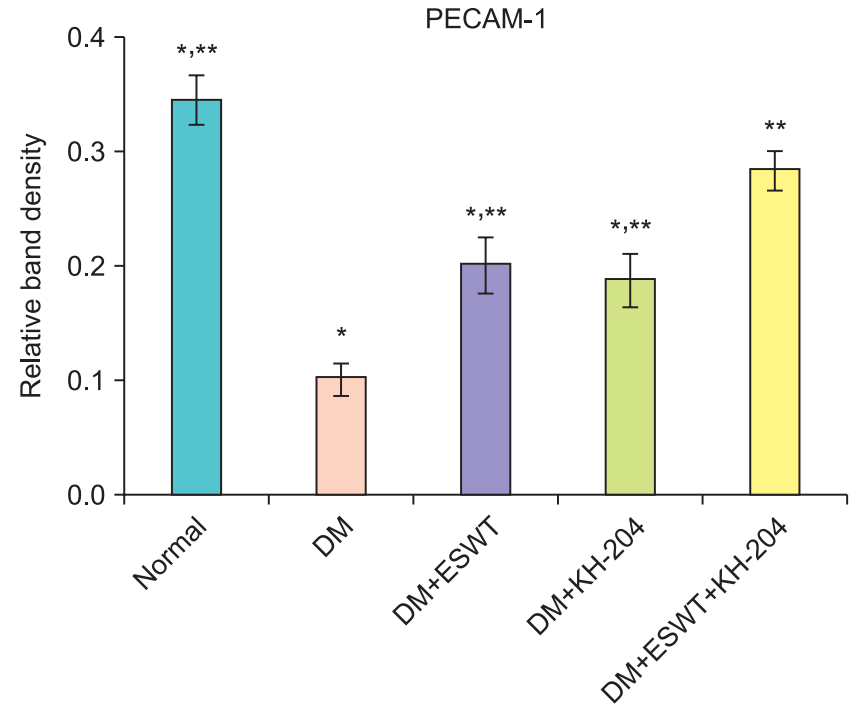

Fig. 5. (A) Comparison of the relative platelet endothelial cell adhesion molecule-1 (PECAM-1)/ $\beta$-actin band densities of the different groups by Western blot. (B) Phosphorylated endothelial nitric oxide synthase (P-eNOS) expression in the corporal tissue of each group assessed by Western blotting. The relative band densities of P-eNOS/eNOS (graph). DM, diabetes mellitus; ESWT, extracorporeal shock wave therapy; KH, Korean herbal formula. Data are expressed as means \pm standard deviations. ${ }^{*}$ Statistical significance in comparison with the DM+ESWT+KH-204 group. ${ }^{* *}$ Statistical significance in comparison with the DM group.

DM induced ED, the endothelial tissue apparently decreased. They hypothesized that the poor blood supply in the corpus cavernosum was the key reason for DM induced ED. Some researchers have found that ESWT increased VEGF in the injured tissue, and thereby improved regeneration of endothelial cells and vascular repair [26]. In addition, we found that the VEGF content in the corpus cavernosum of ED rats was lower in comparison with the control group.

In our previous study, we found KH-204 as an oriental herbal medicine could effectively decrease oxidative stress [17]. Thus, we considered that KH-204 could protect the corpus cavernosum from vascular injury by reducing oxidative stress. In this study, we found that the ICP was higher in the ESWT and KH-204 groups than in the DM group. The vascular endothelium content was also higher in the DM+ESWT+KH-204 group than in the single treatment group. The ICP result showed a similar trend. On the one hand, we thought ESWT could increase VEGF in the corpus cavernosum, improve regeneration of endothelial cells, promote vascular repair, and restore the blood supply. On the other hand, KH-204 could decrease oxidative stress in the corpus cavernosum of DM rats, reduce vascular damage, and protect injured tissue. Thus, we hypothesize that the protection mechanism and recovery mechanism of vascular and nerve regeneration coexist simultaneously, and improve ED.

Qiu et al. [24] and Li et al. [27] found that ESWT increased the expression of nNOS in the corpus cavernosum of DM rats, and they believed nNOS played an important role in regulating the dilation of blood vessels. With regard to this result, we found that, compared with the results of the ESWT group, nNOS had a higher expression in the DM+ESWT+KH-204 group, which also explained the ICP results. The combination of ESWT and KH-204 stimulated the nerve of the penis to produce a large amount of nNOS. That released nNOS combines with surrounding vascular smooth muscle cells, to expand the blood vessels and achieve a normal erection. Wang et al. [28] found that oxidative 
stress could increase cell injury. We also believe that it was the long-term oxidative stress in cavernous tissues that resulted in nerve injury and a reduction in the release of nNOS, which would induce ED. Oxidative stress was effectively inhibited by KH-204, which could reduce nerve injury in cavernous tissues. Thus, adequate nNOS produced by the nerve of the penis could play a role in the erectile response and might be one of the prime reasons that KH-204 improved ED.

In this study, administration of KH-204 and ESWT showed a better influence on ED treatment, but the further interaction between KH-204 and ESWT was not elucidated. The effect of angiogenesis of ESWT and the recovery of vascular injury obtained by the anti-oxidative stress effect of $\mathrm{KH}-204$ are thought to help the recovery of vascular ED cooperatively. In addition, stimulation of endothelial cell by ESWT increase nNOS expression by increasing VEGF expression and anti-oxidative stress of KH-204 may also increase nNOS, which may play a role in synergic effect of combination therapy. The combination therapy of ESWT and KH-204 is expected to show better results in the clinical field than the single treatments because of its synergistic effect.

\section{CONCLUSIONS}

We confirmed the potential efficacy of ESWT and KH204 in the treatment of ED using an animal model. This combination is expected to have good potential clinical results in the future treatment of refractory $\mathrm{ED}$.

\section{CONFLICTS OF INTEREST}

The authors have nothing to disclose.

\section{ACKNOWLEDGMENTS}

This research was supported by a grant of Comprehensive and Integrative Medicine R\&D project through Comprehensive and Integrative Medicine Institute (CIMI), funded by the Ministry of Health \& Welfare (MOHW), Republic of Korea (grant number: 090-091-3000-3038-301-32001).

\section{REFERENCES}

1. Song WH, Park J, Yoo S, Oh S, Cho SY, Cho MC, et al. Changes in the prevalence and risk factors of erectile dysfunction during a decade: the Korean Internet Sexuality Survey (KISS), a 10-year-interval web-based survey. World J Mens Health 2019; 37:199-209.

2. Smith WB 2nd, McCaslin IR, Gokce A, Mandava SH, Trost L, Hellstrom WJ. PDE5 inhibitors: considerations for preference and long-term adherence. Int J Clin Pract 2013;67:768-80.

3. Hatzichristou D, d'Anzeo G, Porst H, Buvat J, Henneges C, Rossi A, et al. Tadalafil $5 \mathrm{mg}$ once daily for the treatment of erectile dysfunction during a 6-month observational study (EDATE): impact of patient characteristics and comorbidities. BMC Urol 2015;15:111.

4. Hazan-Molina H, Reznick AZ, Kaufman H, Aizenbud D. Periodontal cytokines profile under orthodontic force and extracorporeal shock wave stimuli in a rat model. J Periodontal Res 2015;50:389-96.

5. Becker M, Goetzenich A, Roehl AB, Huebel C, de la Fuente M, Dietz-Laursonn K, et al. Myocardial effects of local shock wave therapy in a Langendorff model. Ultrasonics 2014;54:131-6.

6. Hayashi D, Kawakami K, Ito K, Ishii K, Tanno H, Imai Y, et al. Low-energy extracorporeal shock wave therapy enhances skin wound healing in diabetic mice: a critical role of endothelial nitric oxide synthase. Wound Repair Regen 2012;20:887-95.

7. Hatzichristou D. Low-Intensity Extracorporeal Shock Waves Therapy (LI-ESWT) for the treatment of erectile dysfunction: where do we stand? Eur Urol 2017;71:234-6.

8. Vardi Y, Appel B, Jacob G, Massarwi O, Gruenwald I. Can lowintensity extracorporeal shockwave therapy improve erectile function? A 6-month follow-up pilot study in patients with organic erectile dysfunction. Eur Urol 2010;58:243-8.

9. Chung E, Cartmill R. Evaluation of clinical efficacy, safety and patient satisfaction rate after low-intensity extracorporeal shockwave therapy for the treatment of male erectile dysfunction: an Australian first open-label single-arm prospective clinical trial. BJU Int 2015;115 Suppl 5:46-9.

10. Lei H, Xin H, Guan R, Xu Y, Li H, Tian W, et al. Low-intensity pulsed ultrasound improves erectile function in streptozotocin-induced type I diabetic rats. Urology 2015;86:1241.e11-8.

11. Jeon SH, Shrestha KR, Kim RY, Jung AR, Park YH, Kwon O, et al. Combination therapy using human adipose-derived stem cells on the cavernous nerve and low-energy shockwaves on the corpus cavernosum in a rat model of post-prostatectomy erectile dysfunction. Urology 2016;88:226.e1-9.

12. Jeong HC, Jeon SH, Qun ZG, Kim KS, Choi SW, Bashraheel F, et al. Effects of next-generation low-energy extracorporeal shockwave therapy on erectile dysfunction in an animal model of diabetes. World J Mens Health 2017;35:186-95.

13. Peyton CC, Colaco MA, Kovell RC, Kim JH, Terlecki RP. Erectile dysfunction is predictive of endothelial dysfunction in a well visit population. J Urol 2016;195:1045-50.

14. Wu N, Shen H, Liu H, Wang Y, Bai Y, Han P. Acute blood glu- 
cose fluctuation enhances rat aorta endothelial cell apoptosis, oxidative stress and pro-inflammatory cytokine expression in vivo. Cardiovasc Diabetol 2016;15:109.

15. Bae WJ, Ha US, Choi JB, Kim KS, Kim SJ, Cho HJ, et al. Protective effects of $\mathrm{KH}-204$ in the bladder of androgen-deprived rats. World J Mens Health 2015;33:73-80.

16. Choi SW, Jeon SH, Kwon EB, Zhu GQ, Lee KW, Choi JB, et al. Effect of Korean herbal formula (modified Ojayeonjonghwan) on androgen receptor expression in an aging rat model of late onset hypogonadism. World J Mens Health 2019;37:105-12.

17. Jang H, Bae WJ, Kim SJ, Cho HJ, Yuk SM, Han DS, et al. The herbal formula $\mathrm{KH}-204$ is protective against erectile dysfunction by minimizing oxidative stress and improving lipid profiles in a rat model of erectile dysfunction induced by hypercholesterolaemia. BMC Complement Altern Med 2017;17:129.

18. Kim IG, Piao S, Lee JY, Hong SH, Hwang TK, Kim SW, et al. Effect of an adipose-derived stem cell and nerve growth factorincorporated hydrogel on recovery of erectile function in a rat model of cavernous nerve injury. Tissue Eng Part A 2013; 19:14-23.

19. Lu Z, Lin G, Reed-Maldonado A, Wang C, Lee YC, Lue TF. Low-intensity extracorporeal shock wave treatment improves erectile function: a systematic review and meta-analysis. Eur Urol 2017;71:223-33.

20. Kitrey ND, Gruenwald I, Appel B, Shechter A, Massarwa O, Vardi Y. Penile low intensity shock wave treatment is able to shift PDE5i nonresponders to responders: a double-blind, sham controlled study. J Urol 2016;195:1550-5.

21. McMahon C. Efficacy and safety of daily tadalafil in men with erectile dysfunction previously unresponsive to on-demand tadalafil. J Sex Med 2004;1:292-300.

22. Sáenz de Tejada I, Anglin G, Knight JR, Emmick JT. Effects of tadalafil on erectile dysfunction in men with diabetes. Diabetes Care 2002;25:2159-64.

23. Tepeköylü C, Wang FS, Kozaryn R, Albrecht-Schgoer K, Theurl M, Schaden W, et al. Shock wave treatment induces angiogenesis and mobilizes endogenous CD31/CD34-positive endothelial cells in a hindlimb ischemia model: implications for angiogenesis and vasculogenesis. J Thorac Cardiovasc Surg 2013;146:971-8.

24. Qiu X, Lin G, Xin Z, Ferretti L, Zhang H, Lue TF, et al. Effects of low-energy shockwave therapy on the erectile function and tissue of a diabetic rat model. J Sex Med 2013;10:738-46.

25. Gerber RE, Vita JA, Ganz P, Wager CG, Araujo AB, Rosen RC, et al. Association of peripheral microvascular dysfunction and erectile dysfunction. J Urol 2015;193:612-7.

26. Nishida T, Shimokawa H, Oi K, Tatewaki H, Uwatoku T, Abe $\mathrm{K}$, et al. Extracorporeal cardiac shock wave therapy markedly ameliorates ischemia-induced myocardial dysfunction in pigs in vivo. Circulation 2004;110:3055-61.

27. Li H, Matheu MP, Sun F, Wang L, Sanford MT, Ning H, et al. Low-energy shock wave therapy ameliorates erectile dysfunction in a pelvic neurovascular injuries rat model. J Sex Med 2016;13:22-32

28. Wang L, Tian W, Uwais Z, Li G, Li H, Guan R, et al. AGEbreaker ALT-711 plus insulin could restore erectile function in streptozocin-induced type 1 diabetic rats. J Sex Med 2014;11:1452-62. 